\title{
The MetaOPAC Azalai Italiano (MAI): geography and evolution of Italian online catalogues.
}

\author{
Antonella De Robbio*, Caterina Barazia**, Paola Rossi***, Micaela Mezzetto** \\ * Law Libraries, University of Padova, Padova, Italy \\ ** University of Pavia Libraries, Pavia, Italy \\ *** CILEA Consortium, Milan, Italy
}

\begin{abstract}
Purpose: The purpose of this paper is to consider and discuss some data about the evolution of Italian Online Public Access Catalogues (OPACs) in the last decade and the possibility to search them cumulatively through the MetaOPAC Azalai Italiano (MAI). Scholars all over the world are interested in Italian OPACs due to the treasures and the historical documents owned by Italian libraries.

Design/methodology/approach: After a brief introduction on union and virtual catalogues, with special attention to the Italian scenario, Italian OPACs are illustrated with a rich amount of data about their usage, their geographical distribution, and their growth. All data are available on the Web and have been collected in the time through the analysis of log files and the database of the Italian OPACs. Findings: The evolution of Italian OPACs suggests new developments of MAI, like extension of search to some contents of digital libraries. These are studied and, as far as allowed by the available human resources, progressively adopted in order to improve the quality of the meta-search.

Research limitation/implication: As this tool that has no exact parallels in other countries, this article mainly adopts a descriptive and heuristic approach, rather than evaluating MAI in comparison with other services.

Originality/value: Although a wide amount of literature on MAI is available, only few articles are in English. This article then aims to increase the knowledge of MAI for a broadest group of non-Italian readers.
\end{abstract}

Keywords: Online catalogues, Online databases, Libraries, Italy

Paper type: Case study

\section{Introduction}

The historical context of Italy brought to Italian libraries a hugely rich heritage of books of interest to scholars all over the world.

Manuscripts, incunabula, and editions of the following centuries are widely studied for their stylistic and typographic features, and documents from all times have historical interest.

Access to bibliographical data offered by OPACs highly increases the possibilities for worldwide scholars to widen and improve their research.

Unlike other countries, where one important national library or few university libraries own most works of interest to scholars, in Italy even a little library in a small city can own important and rare documents; therefore, it is crucial for users to have a little number of broad coverage OPACs by which most documents can be accessed.

In Italy, library computerization and cooperation have been delayed in comparison with other European countries (Tronchin, 2008), and many libraries still today have no OPAC. The first national cooperation project has been the National Library Service (SBN) [T] (Metitieri and Ridi, 2005). It started in the 1980s with many ambitions, but mainly focused on cataloguing rather than user service. The SBN libraries share cataloguing, working in a hierarchical structure: each library is associated to a 
regional SBN "pole", in which database the records of the participant libraries are collected. All data of the poles are also copied in a national database, the "Index".

The first union OPAC of SBN (the SBN Index) was online only since 1992 [II].

Although the OPAC SBN Index is a good service, covering approximately 3900 libraries out of the about 15000 Italian public libraries (Giordano, 2002), its complex architecture and its little flexibility limit the participation of a larger number of libraries, in particular of small local libraries and of some university libraries, that address their choice to other products.

In 1970s the Italian National Research Council (CNR), with offices across all the Italian territory, developed a serials cataloguing project, the National Union Archive of Serials (ACNP); in the 1980s the University of Bologna joined the project and developed an information retrieval system to allow public online access to this catalogue: the Italian Union Catalogue of Serials was born [III]. Currently, over 2500 Italian libraries use the ACNP database to catalogue their serials. Although it only contains serials, ACNP is considered as the second Italian union OPAC besides the SBN Index.

In this context MAI, MetaOPAC Azalai Italiano [IV], has been developed since 1999 in order to offer an additional cumulative search into all Italian OPACs (De Robbio and Rossi, 2004).

While SBN and ACNP are union catalogues, MAI is a virtual catalogue, a system without its own bibliographic database. It is the result of cooperation between CILEA, a consortium of computer services for universities [V], and AIB, the Italian Library Association [VI]. Mainly addressed to the community of librarians, this system is freely available online, and offers a friendly interface to simultaneous cumulative search (metasearch) in a great number of different Italian OPACs.

A virtual catalogue shows the same bibliographical record several times, one for each database searched. On the other hand, a union catalogue enters the bibliographical data of the participant libraries without duplicates, and shows a single item with all holdings linked (Preece, 2001). The cooperation level is different in the two types of catalogues: a union catalogue (e.g. Melvyl, Copac) needs a greater technical, cooperative, and economical effort. Each participant library inputs its data into a single database (Cousins, 1999).

When it is impossible to build a union catalogue, the virtual catalogue solves the problem to offer a coverage as broad as possible. A virtual catalogue uses an information retrieval language to launch a search query in different databases, collects the results and shows them in a Web page.

An important information retrieval protocol is Z39.50 (NISO, 2003), that allows to search multiple databases supporting the Z39.50 protocol in a single search, to collect data within a common set of indexes, and to show them in a uniform way. The Z39.50 protocol shows some limitations (Coyle, 2000), but the main restriction to its diffusion is the little use of databases that support Z39.50.

Another protocol to retrieve metadata from different databases is OAI-PMH (OAI, 2008), that allows to collect formatted data and to store them in a common index, to which the search queries are sent. The OAI harvester is very common in the context of open archives, but is less used for searching OPACs.

An easy and successful way to search different catalogues is to use the HTTP protocol to send GET URLs conveying search queries to OPACs. It also exists a protocol, the SRU (Search and Retrieve by URL), maintained by the Library of Congress (Library of Congress, 2008).

The success of virtual catalogues is demonstrated by such catalogues as Karlsruher Virtueller Katalog (KVK) [VII], The European Library [VIII], or the recent Cat Cymru (beta version) [IX], buildt by the libraries of Wales (Bevan and Tyler, 2009). KVK allows to access library and book trade catalogs via WWW; The European Library uses a complex architecture of federated search that includes the SRU protocol, the Z39.50 gateway, and OAI metadata harvesting; Cat Cymru uses a commercial software for federated Web search. MAI uses the HTTP protocol to search the Italian catalogues.

\section{The MAI service}

MAI can be accessed from the Italian OPACs section [IV] of AIB-WEB, the AIB website (Barazia and Rossi, 2007). This webspace includes the Directory of Italian OPACs, a project launched by Riccardo Ridi in 1997 on which MAI is founded. 
The directory lists all online catalogues in Italy and neighbouring Italian-speaking areas (Vatican City, Republic of San Marino, Swiss Canton Ticino).

Each OPAC is catalogued in a database accessible through the Web. Each record contains a data set that include OPAC name, type of participant library/ies, links to information web pages about the catalogue and its libraries, geographical coverage, type of materials included and links to access its interfaces.

The directory is mantained by a team of volunteer librarians, who organize the editorial work through a mailing list (aw-opac@aib.it).

The OPACs catalogued in the database that have a set of minimum requirements are then connected by CILEA technical staff to the Azalai software, the search engine software developed by CILEA, in order to allow their simultaneous search [X].

A former project by CILEA, called the Virtual Bibliographic Catalogue of Lombard Universities [X]], uses the Azalai search engine too; its first on-line application dates back to 1997.

The aim of MAI is to provide a metasearch tool as complete as possible, offering comprehensive coverage of the documents owned by the Italian libraries and allowing to collect documents which are otherwise difficult to find in Italy.

Guidelines for the inclusion of OPACs follow the principle not to duplicate information: for example, if a union catalogue includes data from a single library which also has its own single OPAC, the former is connected rather than the latter. The two major Italian union catalogues, SBN and ACNP, are excluded from the metasearch (although in case a regional SBN pole has a local OPAC, this is connected).

Three interfaces enabling access to MAI content have been created:

1. selective search (OPAC search)

2. global search (general metasearch)

3. search by area (regional search)

The selective OPAC search [XII] was the first one created. It allows the user to specify the type of document, the geographical area and the typology of the libraries he is looking for. User can either choose to browse a list of OPACs, on which he can make an exact search, or directly search the selected OPAC. This function is the least used and this is probably because the majority of the people is more attracted by the quickest search.

Global search [X] sends a query to all OPACs (amounting to 336 at January 2009) at the same time. This feature performs the main purpose of MAI metasearch: enabling the retrieval of rare documents with high quickness and synthesis. This kind of search offers complete answers to users, and its layout serves the purpose (40 records per page that can be look through quickly). In case of many documents, this is highly helpful in order to allow users to examine the whole retrieved outcomes: although users cannot avoid non-relevant records, they can browse the list of results. MAI allows to search OPACs even when they are union catalogue.

The search by geographical area [XIII] starts with an active map. This kind of search can be set depending on personal needs. Although at the beginning it can seem quite similar to the selective search, it is different as it allows the user to surf the directory page or to browse a list of OPACs linked to the selected area.

In the last few years, the system implemented a series of features and acquired new resources. In addition, its model allowed the creation of external projects (special metaOPACs).

Special metaOPACs are devoted to disciplinary fields, for example architecture, or to specific kinds of libraries, or to library types in a geographical area. Another instance is the "MAI for NILDE" [XIV]. NILDE is a Document Delivery service created by the CNR to which many Italian libraries are connected. NILDE users are interested in this special MetaOPAC because it searches the OPACs of those libraries which are not included in the main national catalogues, SBN and ACNP. Special metaOPACs are developed in cooperation with institutions or companies which gain the ownership of the MetaOPAC. These organizations host the MetaOPAC pages on their websites, 
whereas data, software, servers and all the technical arrangements depend on MAI/CILEA. Moreover, the special MetaOPACs exploit the data in MAI database.

The owner organizes his own group of libraries promoting special services, enlarging the number of participating libraries and, consequently, enriching the MAI database.

Beside searching, MAI offers a number of information pages for users and communities which are interested in some kind of collaboration: managers of OPACs and library services, managers of OPACs, administrators of OPAC servers, OPAC developers and others (Figure 1) Specifically, the MAI editors mailinglist (aw-opac@aib.it) serves as dynamic tool for communication among the editorial board and between the editors and external people wanting their OPAC to be connected to MAI metasearch. People who cooperate to this connection must pay attention to connection requirements. Among the other available resources, there are the updated list of OPACs connected to MAI and a list of vendors of software used in Italian libraries.

In order to show the impact of the system, usage statistics, monthly and annual reports are also published.

Figure 1 - The "Italian OPACs" home page

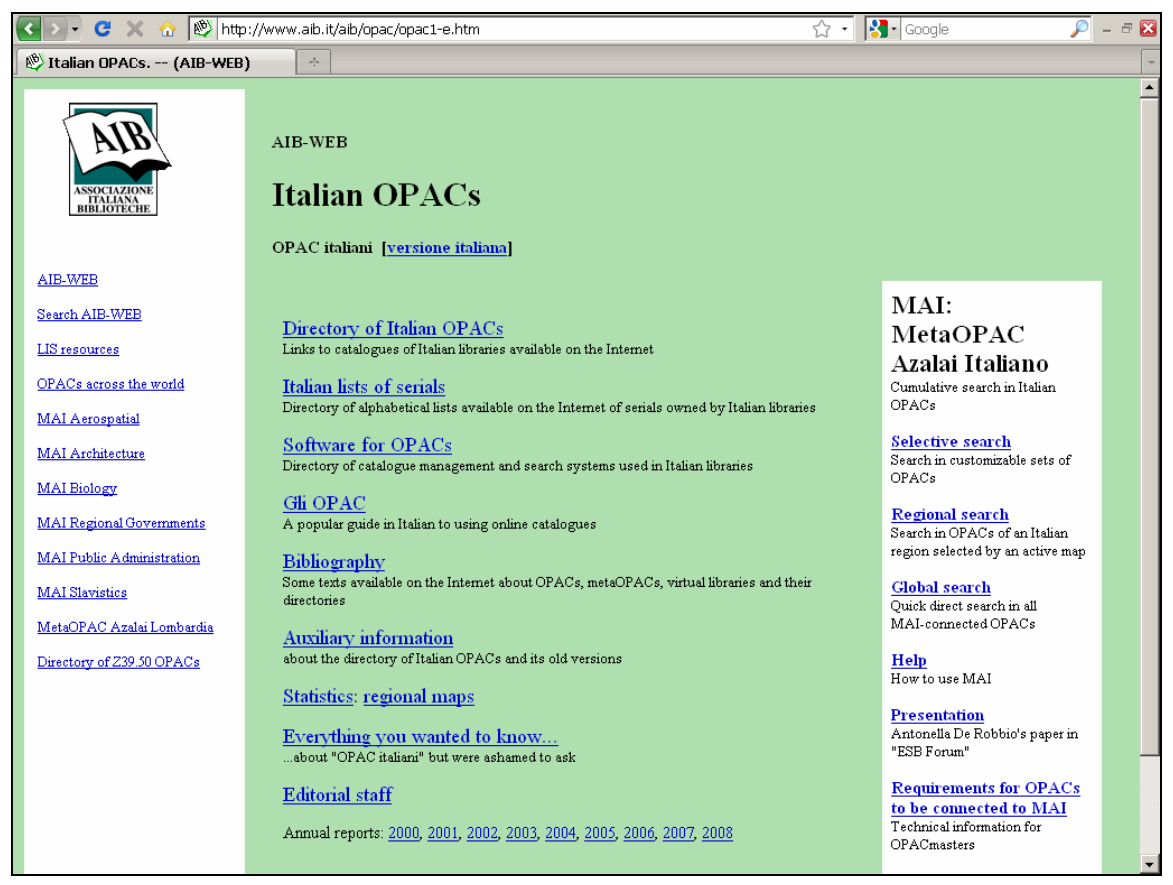

Another service is the Gateway HTTP-Z39.50 [XV]. It supports the web search of OPACs using the Z39.50 protocol (NISO, 2003), and it was set up by CILEA to support MAI metasearch of these particular OPACs. The gateway can search nearly 20 OPACs, and it can be used to query those OPACs Z39.50 which are not included in the service yet.

Since its initial planning stage in 1999 onwards, MAI has always been managed by a cooperation of AIB and CILEA. The agreement, stated in a document visible by the editorial staff, lists all MAI activities, assigning them to AIB and CILEA together, or to AIB and CILEA separately. Even during the years following the planning, mainly devoted to manage the growth of the service, the agreement revealed to be a useful reference point for the organization of the staff activities. As a consequence of the new exigencies and applications - such as special MetaOPAC MAI was interested in - the agreement has been modified and expanded in 2004.

\section{Changes in the Italian OPACs in the period 1999-2008}

In the last few years, the OPACs have shown a significant development. As a consequence, the MAI service has enlarged its databases and improved its features and catchments area (showed in the 
number of accesses to metasearch); furthermore, the MAI data help to investigate the development of OPACs in Italy (De Robbio et al., 2006).

Owing to the diffusion, the change, and the improvement of OPAC services, the metasearch requires a growing care in the management of "connections" (meaning the device that allows Azalai engine to searches the remote OPACs and to receive answers back, on web protocol HTTP).

Between the end of 1999 and the end of 2008, the number of OPACs increased from 326 to 1066, while the number of OPAC connections to MAI metasearch raised from 88 to 336. The following figure (Figure 2) highlights this growth throughout the years.

Although the relation between OPAC services and MAI connections may seem quite simple, there are a wide number of factors which determine it. The MAI metasearch is influenced by both the growth and the variations of OPACs. Furthermore, the MAI metasearch aims at the entirety and at the efficiency achieved through the definition of modalities and application principles which can be easily understandable to the community that managers Italian OPACs.

\section{Figure 2 - Number of OPACs and number of connections to MAI metasearch (1999-2008)}

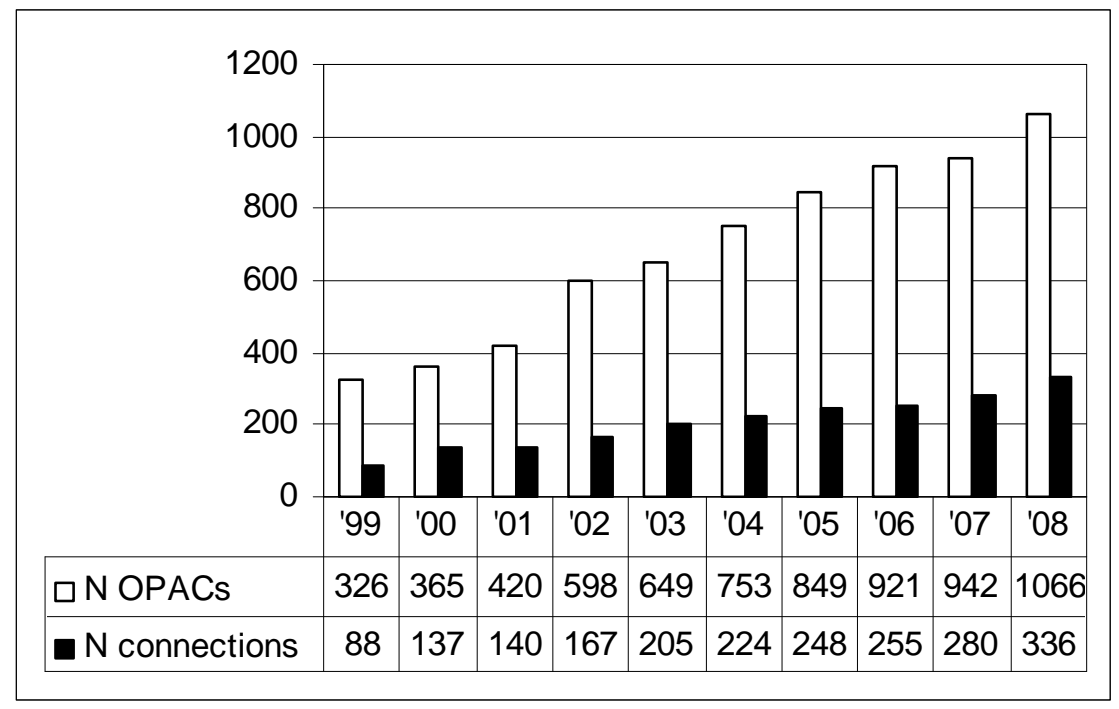

In MAI database each OPAC is catalogued in a record composed of three parts:

1. The $O P A C$, that includes information about OPAC name, type of participant library/ies, links to information web pages about the catalogue and its libraries, geographical coverage.

2. One or more Sections, that include information about the types of materials included.

3. One or more Interfaces, that include the links to the URL of the interfaces, and some technical notes.

Each Section has a related interface; sometimes it can have more than one: a web form, a Z39.50 connection, an outdate search form collateral to the experimental search, etc.. The three parts of each record are connected to Azalai engine.

During the last years many changes occurred to OPACs:

- spread of union catalogues

- merging of single OPACs into union catalogues

- catalogues of special materials merge into union OPACs with the same specialization

- new format standards

- change in OPAC software version; OPAC customization

- use of new architectures for OPACs

- processing of new font sets (Unicode, etc.) 
The medium rate of database changing, for OPAC, section and interface, was the $34 \%$ in 2005 , the $28 \%$ in 2006 , the $27 \%$ in 2007 and the $28 \%$ in 2008.

MAI metasearch connections are related to those OPAC sections which are searchable with a research URL. At the end of 2006 the "distinct" OPAC services connected to MAI are 245, with respect to the total of 255 connections. The connection searching a second section of material are only 10. This datum is a minimum if compared to the data of some years before, pointing that the sectiondivision of OPACs was decreasing. On the other hand, the OPAC services established a unique bibliographic research form, in which the type of document is considered a selection parameter. In the latest years the OPACs have been enriched with on-line documents, e-books, e-journals. The MAI bibliographic research form allows to retrieve also this documents.

As far as OPAC connection is concerned, this are the changes which is essential to point out.

- reducing connections but maintaining the possibility to search all the resources

- enriching the metasearch interfaces with filters based on the type of document

- allowing the search of the OPACs that do not implement it, specifying what to do in such cases and measuring their percentage

- proposing to OPACs managers the inclusion of a filter on the type of document.

The requirements for connecting an OPAC include at least the presence of an author index and a title index. As far as the other seven indexes in MAI search form (object, publisher, time of publication, ISSN/ISBN, series, Dewey Decimal Classification, all fields) are concerned, OPACs provides to MAI those indexes which were implemented depending on their own characteristics and local needs. In the selective search MAI users can see the available indexes percentages (Table 1). The comparison of the indexes over the time allows to analyze their growth in the Italian OPACs.

Table 1-Availability of indexes in MAI research in the years 2003 and 2008.

\begin{tabular}{|l|l|r|}
\hline \multicolumn{1}{|c|}{2003} & \multicolumn{1}{|c|}{ Indexes } & 2008 \\
\hline $76 \%$ & subject & $61 \%$ \\
\hline $53 \%$ & publisher & $68 \%$ \\
\hline $53 \%$ & date & $60 \%$ \\
\hline $21 \%$ & ISSN/ISBN & $43 \%$ \\
\hline $32 \%$ & series & $36 \%$ \\
\hline $38 \%$ & DDC & $52 \%$ \\
\hline $49 \%$ & all fields & $65 \%$ \\
\hline
\end{tabular}

\section{MAI virtual catalogue}

The aim of MAI metasearch is to retrieve as many documents as possible for its users. However, the retrieval of documents have to be the most relevant possible. Sometimes two or more OPACs connectible to MAI can lead to a partial overlapping, and the OPAC that allows to achieve the best result is the only chosen for the connection.

The total number of OPACs in MAI database (December 2008) is 1066 (sections 1337, interfaces 1411), while the total number of connections is 336. Up to this moment, MAI metasearch covers:

- $48,2 \%$ of OPAC services: calculated in unities of connected services with respect to the number of interest services.

- $53,72 \%$ of Web interfaces: calculated in unities of connected interfaces with respect to the whole number of interest interfaces.

With "interest services" we mean those services that really contribute to the virtual catalogue, not the redundant services.

As regards to special MetaOPACs, at the end of 2008 the number of connections was 358. The total number of the general and special connections managed by MAI were 694 . The new connections 
will continue to grow, gradually achieving MAI' s purpose of covering all the owned recovered resources.

The answers to metasearch (positive, void, server not available etc.) present a case study belonging to the final user feedback to the validity of the received answer. Beyond OPACs changes by their OPACs managers, some librarians/OPACs managers could carry out their own comparison controls and reveal mismatches. This information provides a useful collaboration to MAI technical management.

A comparison is given between MAI metasearch and the indications on bibliographic information retrieval by the international standard Z39.50 protocol (NISO, 2003). MAI search meets the Level 0 Searching of Bath layout (Bath Group, 2003), the Basic Bibliographic Search: a search on exact terms such as title, author, and subject and "all fields". At Level 0 MAI adds its own indexes - publisher, publication date, DDC, standard numbers and series. MAI layout corresponds to the more common type of search in Italian OPACs, and it results adequate to the document retrieval feature.

The "geographical covering" was introduced in order to select an OPAC depending on the location of the library. It is based on two main information: the minimum area gathering all the libraries (national, regional, provincial, local covering) and the data of the cities or villages, if they are available. This kind of information is nowadays very common in libraries web pages and many OPACs indicate the libraries which participant to catalogue. The geographical covering of OPACs is known in the $98 \%$ of catalogues without approximation. At the end of 2008 the $45.4 \%$ of Italian cities and villages have at least one OPAC.

The maps of all Italian regions present the distribution of OPACs within cities or villages (Mezzetto and Rossi, 2007). Each municipality has a different color depending on the number of OPACs in its territory. For all the regions a chromatic scale has been used, which divides data in eight different levels: from white, indicating that there is no OPAC on the municipality, to black, indicating that there are more than 13 OPACs. Figure 3 shows the geographical map of Lombardy. the name of the respective municipality with its number of OPACs appears if you move the mouse towards municipial areas.

\section{Figure 3 - map of OPACs in Lombardy}

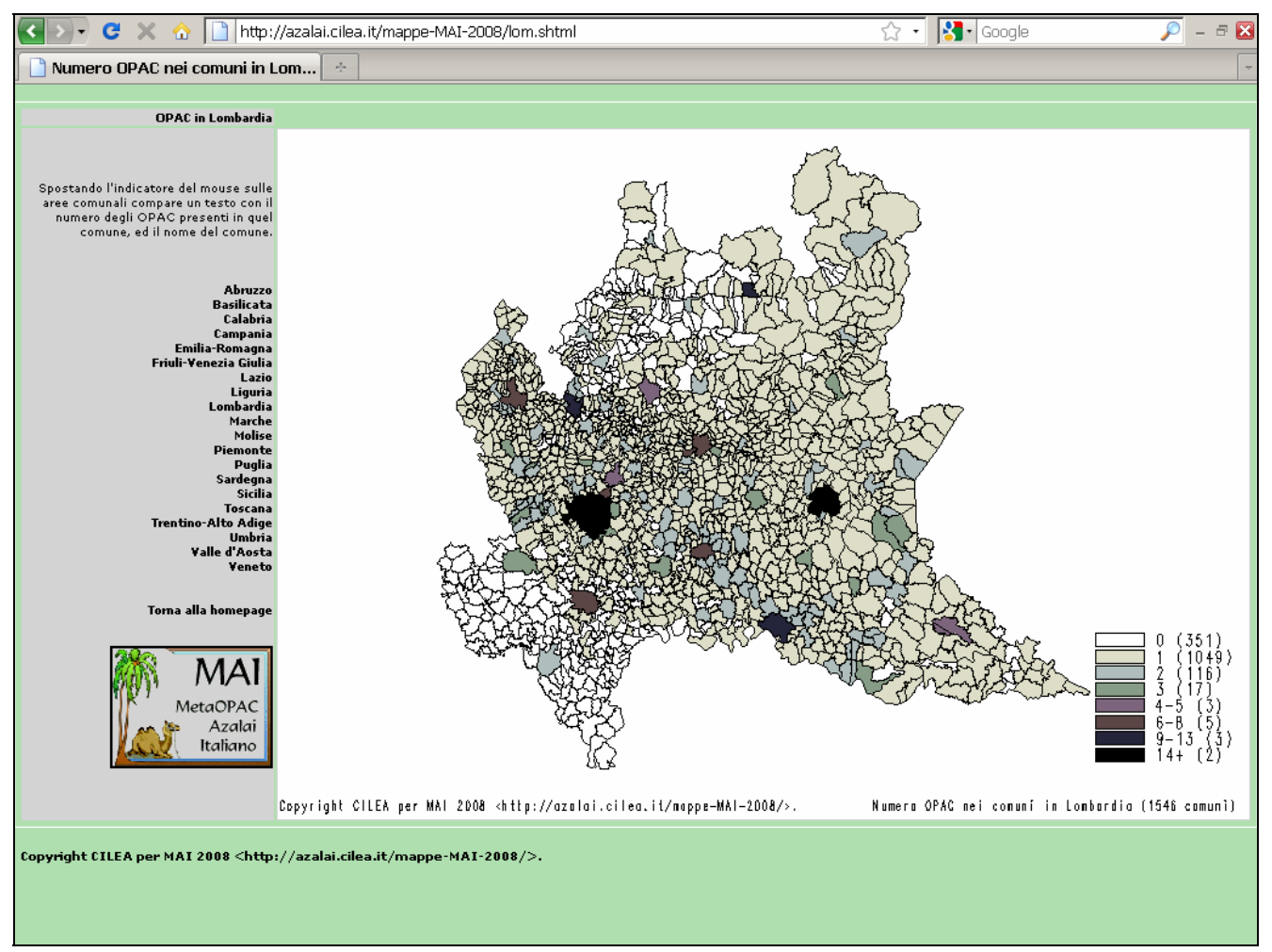


The general overview given by the map is of great interest for MAI management and the related community. It was decided to analyse other descriptive statistics in order to describe the general situation in Italy.

Figure 4 presents in the form of dartboard the regional distribution of OPACs in the 20 Italian regions: moving from the center to the circumference, the regions are ordered on the basis of the growing number of their municipalities. In this second prototype the chromatic scale uses the same classes of regional maps; regions with more than three OPACs are put together with a red label. Regions with the highest number of OPACs per municipalities are those with the less white color.

Figure 4 - regional distribution of $O P A C s$

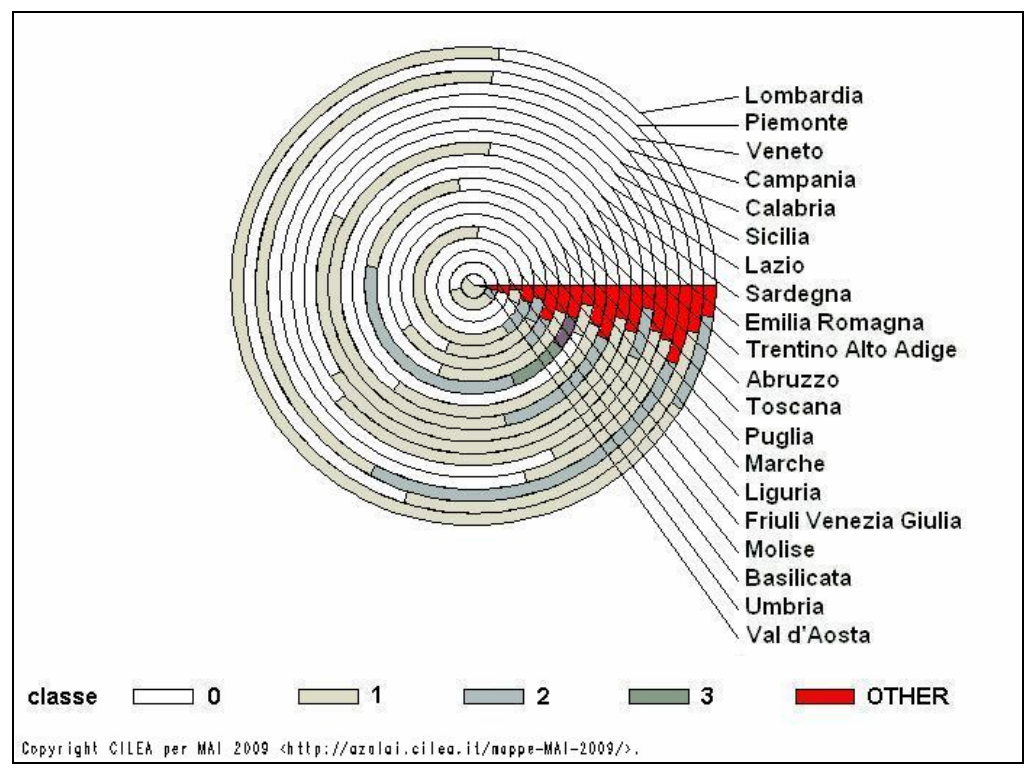

The geographical map of Italy presented in Figure 5expresses graphically the OPACs regional distribution in Italy. For each region, the height of vertical bar indicates the number of municipalities having at least one OPAC in that area.

Figure 5 - Number of Italian municipalities with at least one OP $A C$

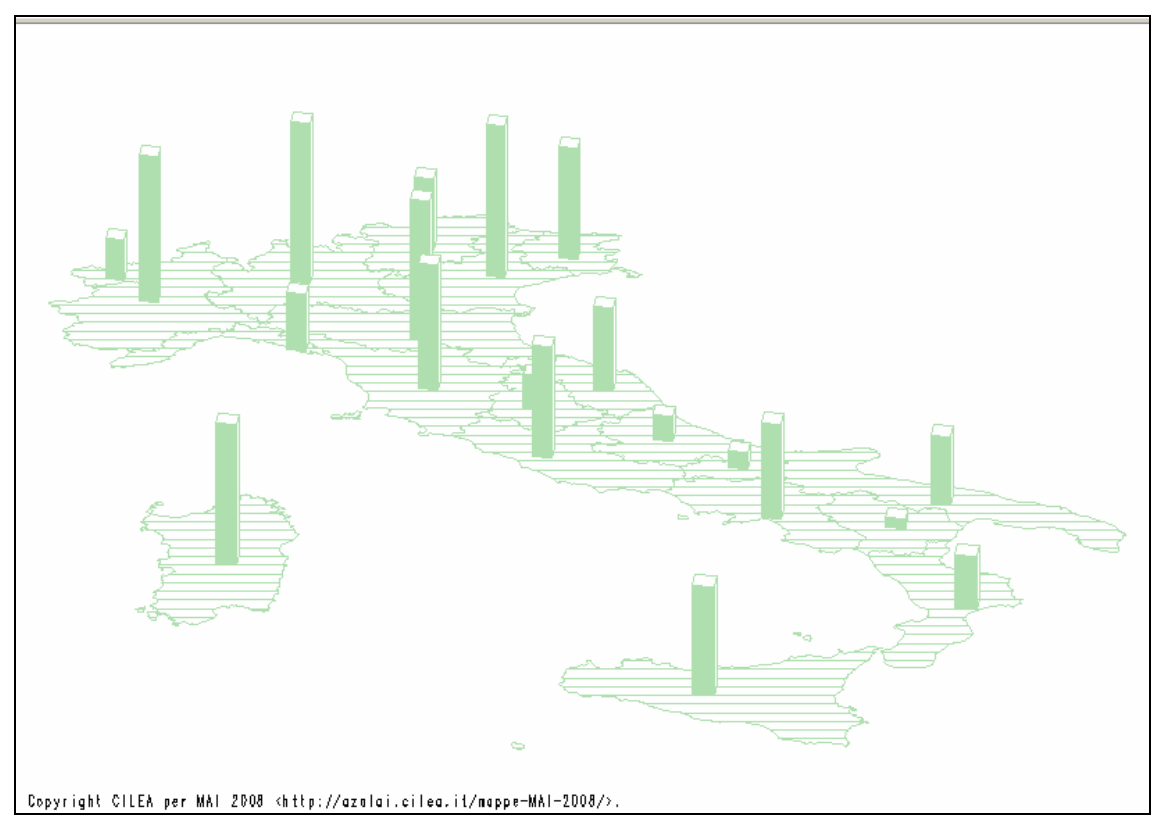


In Figure 6 the vertical bar indicates the relation between the numbers of municipalities having at least one OPAC with respect to the total number of municipalities in that region. As we can see in the following graphic, the bar of the Val d'Aosta region (on top left of the map) is higher: infact, it has a low number of municipalities, but a high OPACs presence.

On average, the regional percentage of OPACs covering in municipalities is $45.4 \%$.

Figure 6 - Number of Italian municipalities with at least one OPAC compared with the number of municipalities

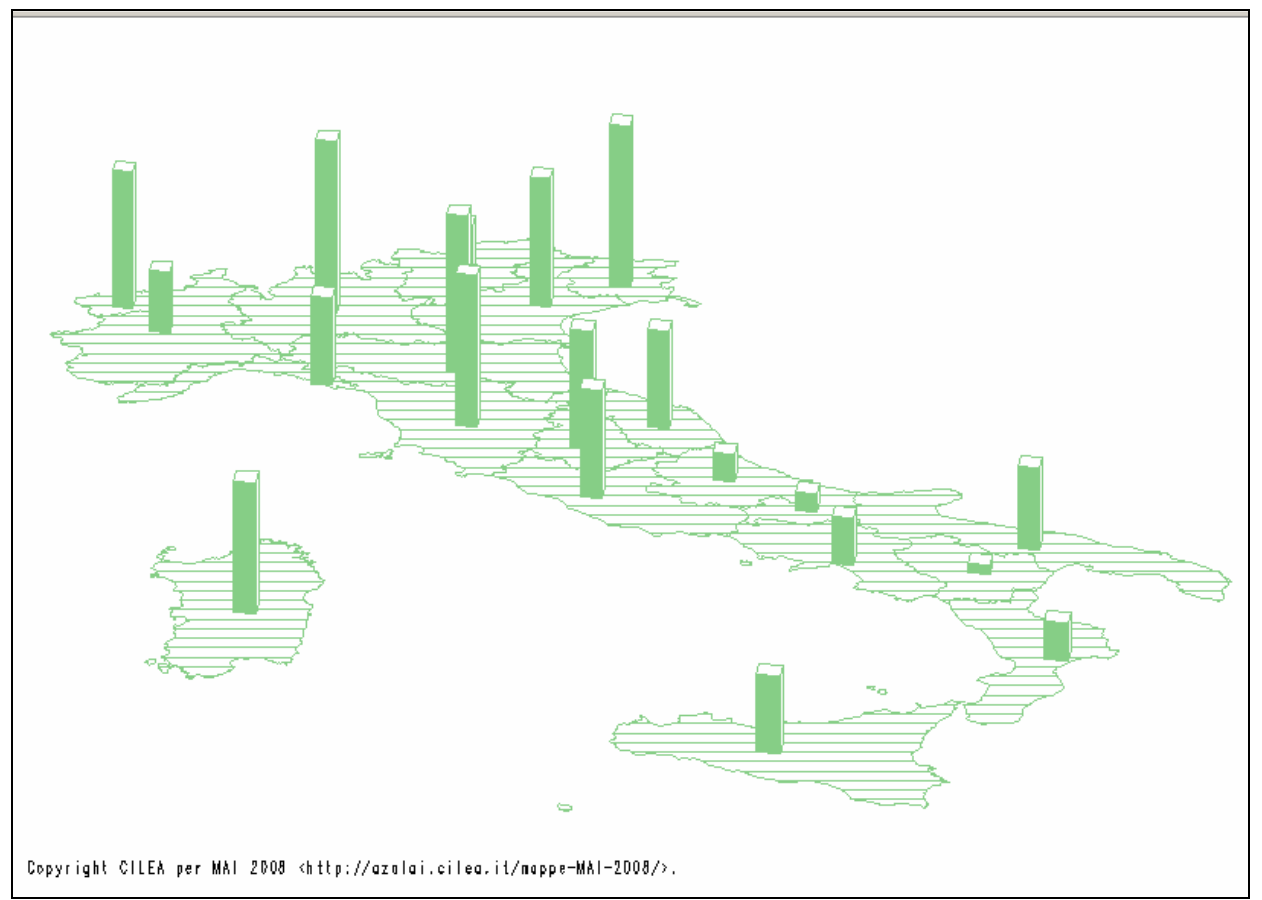

These two maps are accompanied by the data in Table II: the columns entitled "number of municipalities with OPACs' and "presence in regional percentage" contain respectively the data of Figure 5 and Figure 6.

The data of this table have been calculated with statistical tools supplied by SAS [XVI] (SAS software ver. 8.2). 
Table II -OP ACs in Italian municipalities

\begin{tabular}{|l|r|r|r|r|r|}
\hline \multicolumn{1}{|c|}{ region } & $\begin{array}{c}\text { number of } \\
\text { municipalities }\end{array}$ & $\begin{array}{c}\text { number of } \\
\text { municipalities } \\
\text { with OPACs } \\
\text { (presence) }\end{array}$ & $\begin{array}{c}\text { presence in } \\
\text { regional } \\
\text { percentage }\end{array}$ & $\begin{array}{c}\text { ratio of } \\
\text { municipalities } \\
\text { to Italy }\end{array}$ & $\begin{array}{c}\text { ratio of } \\
\text { municipalities } \\
\text { with OPACs to } \\
\text { Italy }\end{array}$ \\
\hline Piemonte & 1209 & 361 & $29,9 \%$ & $14,9 \%$ & $4,5 \%$ \\
\hline Val d'Aosta & 74 & 47 & $63,5 \%$ & $0,9 \%$ & $0,6 \%$ \\
\hline Lombardia & 1546 & 1186 & $76,7 \%$ & $19,1 \%$ & $14,6 \%$ \\
\hline Trentino-Alto Adige & 339 & 120 & $35,4 \%$ & $4,2 \%$ & $1,5 \%$ \\
\hline Veneto & 580 & 443 & $76,4 \%$ & $7,2 \%$ & $5,5 \%$ \\
\hline Friuli Venezia Giulia & 219 & 167 & $76,3 \%$ & $2,7 \%$ & $2,1 \%$ \\
\hline Liguria & 235 & 74 & $31,5 \%$ & $2,9 \%$ & $0,9 \%$ \\
\hline Emilia-Romagna & 341 & 262 & $76,8 \%$ & $4,2 \%$ & $3,2 \%$ \\
\hline Toscana & 287 & 209 & $72,8 \%$ & $3,5 \%$ & $2,6 \%$ \\
\hline Umbria & 92 & 43 & $46,7 \%$ & $1,1 \%$ & $0,5 \%$ \\
\hline Marche & 246 & 101 & $41,1 \%$ & $3,0 \%$ & $1,2 \%$ \\
\hline Lazio & 377 & 154 & $40,8 \%$ & $4,7 \%$ & $1,9 \%$ \\
\hline Abruzzo & 305 & 21 & $6,9 \%$ & $3,8 \%$ & $0,3 \%$ \\
\hline Molise & 136 & 8 & $5,9 \%$ & $1,7 \%$ & $0,1 \%$ \\
\hline Campania & 551 & 115 & $20,9 \%$ & $6,8 \%$ & $1,4 \%$ \\
\hline Puglia & 258 & 80 & $31,0 \%$ & $3,2 \%$ & $1,0 \%$ \\
\hline Basilicata & 131 & 5 & $3,8 \%$ & $1,6 \%$ & $0,1 \%$ \\
\hline Calabria & 409 & 74 & $18,1 \%$ & $5,0 \%$ & $0,9 \%$ \\
\hline Sicilia & 390 & 151 & $38,7 \%$ & $4,8 \%$ & $1,9 \%$ \\
\hline Sardegna & 376 & 217 & $57,7 \%$ & $4,6 \%$ & $2,7 \%$ \\
\hline medium value & $\mathbf{4 0 5 , 0 5}$ & $\mathbf{1 9 1 , 9}$ & $\mathbf{4 2 , 5 \%}$ & $\mathbf{5 , 0 \%}$ & $\mathbf{2 , 4 \%}$ \\
\hline total & $\mathbf{8 1 0 1}$ & $\mathbf{3 8 3 8}$ & & $\mathbf{1 0 0 , 0 \%}$ & $\mathbf{4 7 , 4 \%}$ \\
\hline
\end{tabular}

\section{The usage of MAI}

While Azalai is the Java software for OPACs query enabling above all metasearch and MAI global search, "MAI library" is the whole number of software implementing selective and regional MAI search, database updating, and the production of the directory and data lists.

A new version of MAI library created with the open source software Linux Apache MySQL PHP was released. This "porting" was completed in 2005-2006.

Until 2004, the growth in the use of MAI metasearch [XVII] was about $17 \%$, with a light increase $(+1 \%)$ and a slight drop $(-2 \%)$ in 2006. the use of global search raised $22 \%$ in 2004, and kept this trend until $2007(+3 \%)$, with a drop in $2008(-6,3 \%)$. Global search reached in 2008 the 79,12\% of the total MAI metasearch. The strong development in the use led to the adjustment of the web-farm resources (web farm is the technical solution allowing the running of Azalai). The use of the web farm allowed the improvement of the initial lack of resources: since they had no results, the user repeated the same requests many times, raising the number of the feedback.

The daily average was more than 6000 accesses, but since the usage is more frequent on workdays the data are lower than the real value. MAI charge is not equally distributed during the day: The main peak is between $10 \mathrm{am}$ and $12 \mathrm{am}$, the minor between $4 \mathrm{pm}-6 \mathrm{pm}$ (Figure 7).

Compared with the two major Italian union catalogues, SBN [II] and ACNP [II], MAI meets users approval [ [ $\left.{ }^{1}\right]$.

MAI metasearch drives load to OPACs. On the basis of the previous considerations, MAI managers estimates that the daily load driven to OPACs is higher than the daily average - a useful value to people who set OPACs resources and who are interested into MAI. 
Figure 7 - requests to MAI per hour (May 2007)

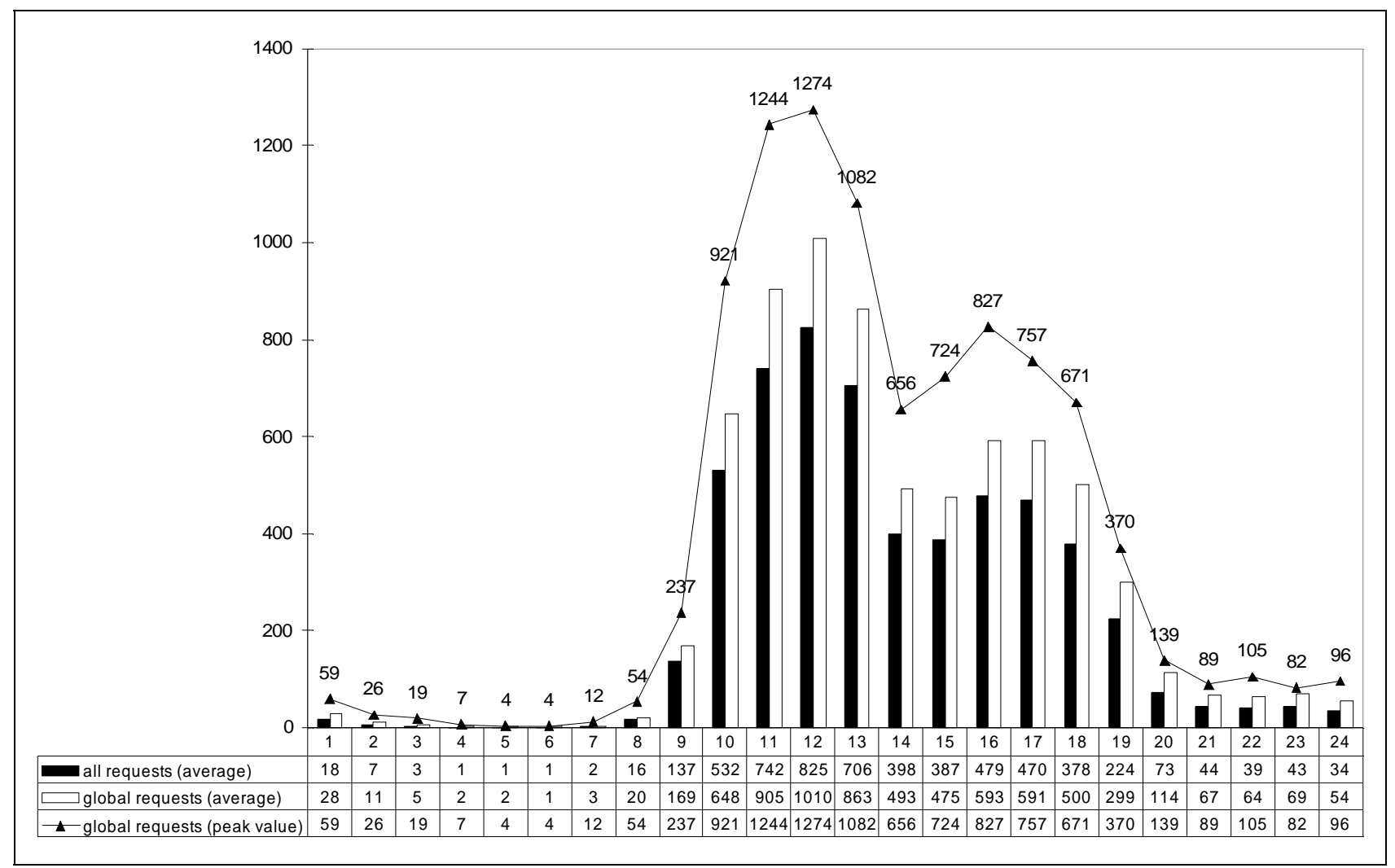

MAI's growth in the number of accesses caused problems of OPACs overloading, so much that in 2005 it requirements of applicability in addition to those of compatibility of OPACs software to MAI server were signalled to the managers of OPACs. Even though the load curve is the same for the systems of OPACs and MetaOPACs, in case of troubles with the OPAC the MAI has its own strategy. It immediately disconnects the OPAC after receiving a communication of overload by the manager of OPAC because it is more important to offer a service to the local users rather than to provide metasearch services to MAI users.

The MAI's planning took advantage of its strong growth to achieve more refined features suitable to the reached levels. This consideration is at the basis of the development of MetaOPAC systems applied to disciplinary, territorial or typology areas. They are used by the specialists of these areas and their usage is moderate with respect to other MAI search. Of the dozen MetaOPAC applications, the most searched are:

- Lombard Public Libraries System: more than 1600 accesses per month; it is active from the Lombardy Region website;

- Educational Libraries: about 700 accesses per month.

- MAI Architecture: about 400 accesses per month.

\section{Conclusions}

The growth in the number of daily users of MAI with the years demonstrates that librarians and libraries users regard the service as an important tool. MAI allows users to find rare documents in Italian libraries with an easy, simultaneous search. Librarians use MAI as a tool for document delivery and inter-library loan services, but also use it as a support in cataloguing and in reference service.

The community of Italian librarians has expressed its interest for possible MAI applications by asking for custom-designed metaOPACs, in order to meet particular needs. The existent special 
metaOPACs were not planned at the birth of MAI. Associations and groups of libraries interested in MAI applications have commissioned new metaOPACs, and still ask for more.

The possibility to have its own OPAC searchable through MAI has encouraged librarians to ask OPAC developers to make their products fitting with MAI technical requirements. The collaboration among MAI staff, librarians, and computer scientists increases through the time; contacts rapidly increase, allowing to become aware of new technical problems and cope with them.

Statistical data available through MAI during a decade allow to obtain information about the growth and the geographical distribution of OPACs in Italy, their technical changes over time, their usage, the types of materials they include. All data enable a global survey of Italian OPACs considered in a national perspective.

The strenght of MAI service is the easy collaboration between staff members: volunteer librarians catalogue OPACs in the MAI database, everyone being responsible for the OPACs of a particular area; CILEA technical staff works independently, caring for OPAC connections and technical issues.

MAI does not receive any funding nor sponsorship. The CILEA consortium cover operating costs of the service (server, technical staff) and has a small income by selling special metaOPACs. Lack of funds is certainly a limitation for the technological progress of MAI. However, the enthousiastic participation of the editors and the commitment of the technical staff have made many improvements to the service possible, especially concerning the development of the search interface. Another direction of research concerns the technical testings for the connection of OPACs to the meta-search: in 2005 the CILEA Gateway HTTP-Z39.50 was designed to search Z39.50 OPACs. In the future, new solutions could be considered to search OPACs using other protocols, such as OAI.

Another interesting topic for staff discussion concerns digital libraries and the possibility of including them in MAI meta-search. Indeed, MAI is originally designed to search OPACs, while digital libraries include a number of very different services, often with characteristics different than OPACs. However, digital libraries that contain documents similar to bibliographic material are very interesting for library users. A user might thus be interested in the possibility of searching simultaneously an OPAC and a digital library that contain the same book, respectively in printed and digital versions. An attractive solution would be to build a meta-OPAC for search in digital libraries, but this would require more resources than those currently available. It has been considered instead the opportunity to include some digital libraries in the MAI database, by describing them in an appropriate way, in order to get closer to the user wishes.

Finally, the publication of English information about MAI and Italian OPACs is expected to reach a broader range of potential users interested in documents of Italian libraries.

\section{References}

Barazia, C., Rossi, P. (2007), "Italian OPACs: a tool to retrieve documents in Italian libraries", ISGL Bulletin: The annual newsletter of the Italian Studies Library Group, Vol. 6, pp. 24 -29, also available at: http://eprints.rclis.org/archive/11459/ (accessed 7 August 2009).

The Bath Group (2003), “The Bath Profile: An International Z39.50 Specification for Library Applications and Resource Discovery. Internationally Registered Profile ISO TC 46 SC 4, February 2004", Release 2.0, Bath Profile Maintenance Agency Library and Archives Canada, avilable at http://www.collectionscanada.gc.ca/bath/tp-bath2-e.htm\#a (accessed 7 August 2009).

Bevan, P. and Tyler, A. (2009), "An integrated library platform: Wales' approach to delivering digital information and resources nationally", Program: electronic library and information systems, Vol. 43 No. 1, pp. 36-48.

Cousins, S. (1999), "Virtual OPACs versus union database: two models of union catalogue provision", The Electronic Library, Vol. 17 No. 2, pp. 97-103.

Coyle, K. (2000), "The Virtual Union Catalog : a comparative study", D-LIB Magazine, Vol. 6 No. 3 , available at: http://www.dlib.org/dlib/march00/coyle/03coyle.html. (accessed 10 August 2009).

De Robbio, A., Gnoli, C. and Rossi, P. (2006), "Tendenze evolutive degli OPAC italiani visti attraverso il MAI", Bollettino AIB (Associazione Italiana Biblioteche), Vol. 46, pp. 69-78. 
De Robbio, A. and Rossi, P. (2004), "MAI : MetaOPAC Azalai Italiano", Program : electronic library and information systems, Vol. 38 No. 2, pp. 97-109, also available at: http://eprints.rclis.org/1169/1/emerald-MAI.pdf. (accessed 10 August 2009).

Giordano, T. (2002), "Library co-operation on ICT in Italy: an overview", Program: electronic library and information systems, Vol. 36 No. 3. pp. 144-151.

Library of Congress (2008), "SRU Search/Retrieval via URL standard", Ver 1.2, available at http://www.loc.gov/standards/sru/ (accessed 10 August 2009).

Mezzetto, M. and Rossi, P. (2007), "MetaOPAC Azalai italiano: mappe geografiche della distribuzione degli OPAC in Italia", Bollettino del CILEA, Vol. 106, pp.16-19, available at: http:/ / bollettino.cilea.it/include/getdoc.php?id $=1403 \&$ article $=622 \&$ mode $=p d f($ accessed 7 August 2009).

National Information Standards Organization - NISO (2003), "Information Retrieval (Z39.50): Application service definition and protocol specification (ANSI/NISO Z39.50-2003)", available at: http://www.loc.gov/z3950/agency/Z39-50-2003.pdf (accessed 10 August 2009).

OAI executive and OAI technical commitee (2008), "The Open Archives Initiative protocol for metadata harvesting", Ver. 2.0, available at: http://www.openarchives.org/OAI/openarchivesprotocol.html (accessed 10 August 2009).

Preece, B. (2001), "Union and virtual catalogs in a consortial environment: edited by Thomas A. Peters", The Journal of Academic Librarianship, Vol. 27 No. 6, pp. 470-472.

Tronchin, L. (2008), "I primi OPAC", in Tronchin, L., I catalogbi elettronici delle biblioteche : tendenze evolutive degli OPAC : tesi di laurea in Biblioteconomia, Corso di laurea in Conservazione dei beni culturali, Facoltà di Lettere e Filosofia, Università Ca' Foscari di Venezia, A.A. 2006-2007, relatore Ridi, R., correlatore Elenteri, P. [Dissertation], available in: ESB forum, http://www.burioni.it/forum/tronchin/ (accessed 7 August 2009).

\section{Standalone URLs}

[1] National Library Service (SBN), http://www.iccu.sbn.it/genera.jsp?s=5\&l=en (accessed 7 August 2009).

[II] The SBN Catalogue http://www.internetculturale.it/moduli/opac/opac.jsp?s=4\&l=en (accessed 7 August 2009).

[III] Italian Union Catalogue of Serials (ACNP) http://acnp.cib.unibo.it/cgi-ser/start/en/cnr/fp.html (accessed 7 August 2009).

[IV] Italian OPACs section - AIB-WEB http://www.aib.it/aib/opac/opac1-e.htm (accessed 7 August 2009).

[V] Lombard Interuniversity Consortium for Automatic Computation (CILEA). http://www.cilea.it/index.php?id=home\&L=1 (accessed 7 August 2009).

[VI] AIB-WEB, the website of the Italian Library Association. http://www.aib.it/aib/aib-e.htm3 (accessed 7 August 2009).

[VII] KVK: Karlsruher Virtueller Katalog http://www.ubka.uni-karlsruhe.de/hylib/en/kvk.html (accessed 10 August 2009).

[VIII] The European Library http://search.theeuropeanlibrary.org/ (accessed 10 August 2009).

[IX] Cat Cymru (beta) http://library.wales.org (accessed 10 August 2009).

[X] Italian Azalai MetaOPAC (MAI). Cumulative query in Italian Internet library catalogues (Global search) http://www.aib.it/aib/opac/mai2.htm3 (accessed 7 August 2009).

[XI] Virtual Bibliographic Catalogue of Lombard Universities, http://azalai.cilea.it/lombardo/ (accessed 7 August 2009).

[XII] Italian Azalai MetaOPAC (MAI). Cumulative query in Italian Internet library catalogues (Selective search) http://www.aib.it/aib/opac/mai.htm3 (accessed 7 August 2009).

[XIII] Italian Azalai MetaOPAC (MAI). Cumulative query in Italian Internet library catalogues (Regional search) http://www.aib.it/aib/opac/mai3.htm3 (accessed 7 August 2009).

[XIV] Network Inter Library Document Exchange NILDE. MetaOPAC, http://azalai.cilea.it/nilde/ (accessed 7 August 2009). 
[XV] Gateway CILEA HTTP-Z39.50, http://gwz.cilea.it/ (accessed 7 August 2009).

[XVI] SAS, http://www.sas.com// (accessed 7 August 2009).

[XVII] Italian Azalai MetaOPAC (MAI). Statistics, http://azalai.cilea.it/statistiche.htm (accessed 10 August 2009).

\section{Further reading}

A comprehensive bibliografy on MAI is available on the AIB-WEB site: Bibliografia: Alcuni testi su $O P A C$, metaOPAC, biblioteche virtuali e relativi repertori = Bibliography on OPACs: some texts on OPACs, metaOPACs, virtual libraries and related directories, Italian Library Association (AIB), http://www.aib.it/aib/opac/op1bib.htm (accessed 7 August 2009).

\footnotetext{
${ }^{1}$ The usage data of SBN compared to access to MAI revealed a proportion of 6,7 for SBN with respect to MAI (May 2004 April 2005). For a more recent comparison, we used the data of the OPAC SBN Gateway on general statistics, which was considered the most suitable source by SBN team. The proportion in 2006 was of 6.4; while at the beginning of 2007 was of 5.9. With respect to ACNP the usage proportion (data March 2006 - February 2007) of ACNP to MAI was 1.9.
} 